\title{
Carbon Nanotube Tip Probes: Stability and Lateral Resolution in Scanning Probe Microscopy and Application to Surface Science in Semiconductors
}

\author{
Cattien V. Nguyen ", Kuo-Jen Chao", Ramsey M.D. Stevens", Lance Delzeit, Alan \\ Cassell", Jie Han", and M. Meyyappan
}

NASA Ames Research Center, MS 229-1, Moffett Field, CA 94035

\begin{abstract}
In this paper we present results on the stability and lateral resolution capability of carbon nanotube (CNT) scanning probes as applied to atomic force microscopy (AFM). Surface topography images of ultra-thin films (2-5 $\mathrm{nm}$ thickness) obtained with AFM are used to illustrate the lateral resolution capability of single-walled carbon nanotube probes. Images of metal films prepared by ion beam sputtering exhibit grain sizes ranging from greater than $10 \mathrm{~nm}$ to as small as $-2 \mathrm{~nm}$ for gold and iridium respectively. In addition, imaging stability and lifetime of multi-walled carbon nanotube scanning probes are studied on a relatively hard surface of silicon nitride $\left(\mathrm{Si}_{3} \mathrm{~N}_{4}\right)$. AFM images of $\mathrm{Si}_{3} \mathrm{~N}_{4}$ surface collected after more than 15 hrs of continuous scanning show no detectable degradation in lateral resolution. These results indicate the general feasibility of CNT tips and scanning probe microscopy for examining nanometer-scale surface features of deposited metals as well as non-conductive thin films. AFM coupled with CNT tips offers a simple and nondestructive technique for probing a variety of surfaces, and has immense potential as a surface characterization tool in integrated circuit manufacturing.
\end{abstract}

- ELORET Corporation

- Charles Evans \& Associales 810 Kifer Road. Sunnyvale. CA 94086-5203

$'$ crnguyen (a) mail.arc.nasa.gov 


\section{Introduction}

Since lijima' discovered carbon nanotubes (CNT), researchers studying these nanometerscale structures and their extraordinary electrical and mechanical properties have proposed many possible applications. Some potential uses of CNTs include field emitters, sensors, tips for scanning probe microscopy, molecular wires and high strength composites. One of the most promising and near-term applications of CNTs is as tips for scanning probe microscopy ${ }^{2}$. The high aspect ratio of CNTs with intrinsic diameter as small as $0.5 \mathrm{~nm}$ and length extending to several microns makes them ideal as scanning probe tips. Since the diameter of the scanning probe tip determines the imaging resolution, CNT tips would offer high resolution, while the length of CNT tips permits the tracing of rough surfaces with steep and deep features. Furthermore, the extraordinary strength and the ability to retain structural integrity after deformation (elastically buckling) make CNT scanning probes very robust, thus resulting in relatively long wearing probes in comparison to conventional silicon scanning probes.

Various approaches exist to attach CNTs to the tip of a scanning probe. The first technique developed by Dai et al. ${ }^{2}$ involves attaching a bundle of multi-walled carbon nanotubes (MWNTs) to the tip of a silicon probe in the AFM cantilever using a polymeric adhesive. Wong et al. ${ }^{3}$ demonstrated that single-walled carbon nanotube (SWNT) can be grown directly onto the tip of silicon scanning probe by chemical vapor deposition (CVD) at $750^{\circ} \mathrm{C}$ using ethylene gas as the carbon feedstock. Nakayama et al ${ }^{4}$ fabricated nanotube probes of various lengths using electrostatic attraction, welding and carbon deposition. Stevens et al. ${ }^{5}$ developed a technique for attaching MWNTs that involved applying a DC field between the CNT source and the tip of silicon scanning probe. This technique appears to be the simplest and most useful for producing a MWNT probe. On the other hand, the direct growth of SWNT tips by CVD techniques offers perhaps the most practical means for producing SWNT scanning probes which by the nature of the CVD process can be scaled up for mass production.

CNT tips, with their diameter in the nanometer-scale, in conjuction with AFM offer a simple, nondestructive and noninvasive technique to characterize features in the 
nanometer realm. As features in semiconductor devices continue to decrease, there is a tremendous need for new surface characterization techniques. For example, as gate dielectrics layer becomes thinner, with the estimation that it will be in the tens of atomic layers by the end of this decade, conventional characterization techniques will not be adequate. In fact AFM may be the best solution for examining surface morphology and uniformity of coverage of nonconducting ultra-thin films. Scanning electron microscopy (SEM) as well as scanning tunneling microscopy will not work on nonconducting samples, and transmission electron microscopy would be a destructive and costly technique. SEM requires that a nonconductive sample be coated with a conductive layer which itself would modify the surface of the thin film to be examined. Additionally, atomic force microscopes operate in ambient environments, and the absence of a vacuum requirement would result in higher throughput. Indeed, there have been recent studies demonstrating the utility of AFM as a surface characterization tool. Wu and Wallace ${ }^{6}$ studied microroughness of polymer thin films by AFM and total-reflection $x$-ray fluorescence (TXRF). The results showed a type of roughness characterized by sharp parallel ridges on an otherwise unadulterated surface which was detectable only by AFM but was not readily apparent in the TXRF spectra. Reynolds and Taylor ${ }^{7}$ showed depthdependent sidewall roughness on the positive-tone resists APEX-E and UV5 using AFM. Strausser $\mathrm{et}^{\mathrm{a} l^{8}}$ studied the evolution of surface morphology of polysilicon by AFM and was able to correlate the sensitivity of surface morphology to growth temperature and pressure. Edrei et al ${ }^{9}$ obtained quantitative and qualitative morphology information of amorphous and polysilicon layers deposited on silicon wafers. In addition to semiconductor surface characterization, CNT probes have many applications in the emerging field of nanotechnology as tools for both characterization and manipulation.

Herein we present results from our investigation into the lifetime stability and resolution of CNT tips as applied to AFM for imaging various surfaces. Representative surfaces of silicon nitride $\left(\mathrm{Si}_{3} \mathrm{~N}_{4}\right)$, iridium and gold thin films have been examined. Images of 280 $\mathrm{nm}$ line/space pattern consisting of $300 \mathrm{~nm}$ thick polymeric resist on a silicon substrate acquired with a MWNT probe are also presented to demonstrate application of CNT probes in metrology. Our results demonstrate the superior performance of CNT probes for AFM and its application as a characterization tool in integrated circuit manufacturing. 


\section{Experimental}

MWNTs were attached to the tips of silicon cantilevers by applying a DC field between the cantilever and MWNT source according to previously published technique ${ }^{10}$. SWNT tip probes were fabricated by a modified chemical vapor deposition technique as follows. Silicon pyramid on AFM cantilevers (Force Modulation Etched Silicon Probe, $\mathrm{k}=0.5-5$ $\mathrm{N} / \mathrm{m}$, fo $=65-80 \mathrm{kHz}$, Digital Instruments, Santa Barbara, $C A$ ) were dip-coated in a liquid catalyst solution" (1.2 $\mathrm{g} \mathrm{AlCl}_{3}, 0.57 \mathrm{~mL} \mathrm{SiCl}_{4}, 90 \mathrm{mg} \mathrm{FeCl}, 6 \mathrm{mg} \mathrm{MoCl}_{2} \mathrm{O}_{2}$ and $1 \mathrm{~g}$ of P123 block copolymers in $15 \mathrm{~mL}$ of ethanol), leaving catalyst islands on the base of the pyramid and along its sides. The coated cantilevers were then heated in air at $500^{\circ} \mathrm{C}$ for two hours in a quartz tube. The temperature was then raised to $700^{\circ} \mathrm{C}$ and sustained in air for an additional 20 minutes. Argon was then passed through the quartz tube and the temperature was raised to $900^{\circ} \mathrm{C}$. Once the temperature reached $900^{\circ} \mathrm{C}$, gas flow was switched to methane (at a flow rate of $1,000 \mathrm{sccm}$ ) for 10 minutes and then the furnace was cooled to room temperature with $1,000 \mathrm{sccm}$ flow of Ar.

A Molecular Imaging PicoScan scanning probe microscope was utilized (in the force vs. distance mode) to characterize the CNT probes as well as to shorten the CNT tips. Images were acquired using Digital Instrument Dimension 5000 Scope. Typical settings for both imaging and characterization were set with a free amplitude of $1 \mathrm{~V}$ and a set point at about $0.7 \mathrm{~V}$. Both MWNT and SWNT tips were shortened by an in situ AFM technique ${ }^{2}$. CNTs were oxidatively shortened in air by applying a DC bias between the tip and silicon substrate (7-15 V) while monitoring in force vs. distance mode (for further details see Results and Discussion section). Metal films used in the study were deposited on mica using an ion-beam sputterer (VCR Group Incorporated model IBS/TM200s) under high vacuum with $2 \mathrm{~mA}$ current and a driving voltage of $8 \mathrm{kV}$. 


\section{Results and Discussion}

Figure 1 shows force versus distance curves from tapping-mode atomic force microscopy (TMAFM). Both amplitude (curve a) and deflection (curve b) signals characteristic of a SWNT tip are shown. The buckling signature of a SWNT tip, resulting from the AFM cantilever pushing into the surface of the substrate, is indicated by a fluctuation in the amplitude versus distance signal. Conventional silicon cantilevers without NT tips do not display such unusual behavior in amplitude, but rather would exhibit a linear dampening to zero amplitude, at which point the deflection signal would instantaneously increase (data not shown). However, the deflection versus distance signal for a cantilever with a SWNT tip remains flat when the tip comes in contact with the substrate, as evident by the decreasing amplitude signal. The deflection signal remains unchanged as the SWNT tip continues to push farther into the substrate. Only until the cantilever pushes pass the NT tip and the silicon tip itself comes in contact with the surface, then the deflection signal increases. The Z-distance from the point where the amplitude is first dampened to the point where the deflection signal begins to increase equals the length of the SWNT tip extending beyond apex of the silicon tip; the NT tip in Fig. 1 shows about $70 \mathrm{~nm}$ length. Due to the flexibility of SWNTs, the SWNT tips need to be shortened to a length less than $100 \mathrm{~nm}$ in order to obtain good quality images. TMAFM force versus distance curves offer an in situ technique to examine CNT tips and to monitor the CNT length during the shortening process.

A SWNT scanning probe tip with its small diameter in contact with the surface affords higher resolution images as compared to that obtained with a larger diameter MWNT scanning probe tip. However a MWNT, consisting of multiple layers of concentric nanotubes, is much stiffer than SWNT and as such MWNT tips can have longer length (up to a few microns) and still be able to produce high quality images. The high aspect ratios of a MWNT tip allow it to trace a rough surface with deep features, which has important implications in metrology. Figure 2 demonstrates this point clearly by comparing AFM images acquired with a regular silicon probe and a MWNT probe. A $280 \mathrm{~nm}$ line/space pattern of polymeric photoresist (300 nm thickness) on silicon substrate has been examined. The AFM image acquired with a regular silicon probe 
shows sloping sides for the resist lines which can be easily explained as artifacts derived from the pyramidal shape of the probe. The image of the same polymeric pattern acquired with a micron long MWNT probe shows no detectable artifact due to tip size and shape. The image scanned with the MWNT tip exhibits photoresist lines with nearly vertical walls, which is the real feature as confirmed by SEM (data not shown). The dramatic difference in these two images clearly illustrates the advantage of MWNT probes over that of conventional silicon probes. It is therefore easy to conclude that MWNT probes have immense potential in metrology as the critical dimension of semiconductor devices becomes smaller.

Another comparison between a conventional silicon probe and a MWNT probe has been made by collecting time-lapse images of a relatively hard surface of a silicon nitride film. The images compared in Fig. 3 clearly demonstrate the stability of a MWNT tip attached to a scanning probe. After continuous scanning for more than 15 hours, the same NT tip probe exhibits no detectable degradation in the lateral resolution of the $\mathrm{Si}_{3} \mathrm{~N}_{4}$ grains, which remain the same small sizes of about $-10 \mathrm{~nm}$. However, after about 12 hours of continuous scanning the lateral resolution of the conventional silicon probe degraded, as generally expected from the wearing down of the silicon tip. The wom-out tip results in an increase in the overall grain sizes and in this particular instance also exhibits an artifact of double images. Clearly, MWNT probes are superior to conventional silicon probes in terms of stability. As characterization tools in semiconductor fabrication, CNT probes would require less frequent changing of probes, which would translate into higher throughputs.

For applications requiring higher lateral resolution imaging, SWNT probes can routinely resolve sub- $5 \mathrm{~nm}$ features. Figure 4 contains three examples of AFM images for a variety of thin film surfaces acquired with a single SWNT probe. The same hard, nonconducting $\mathrm{Si}_{3} \mathrm{~N}_{4}$ surface used above in the stability study with MWNT probes was also imaged with a SWNT tip (Fig. 4a). Clearly, the SWNT tip affords a higher lateral resolution with grain sizes as small as $3 \mathrm{~nm}$. This result indicates that the larger $10 \mathrm{~nm}$ grain size for $\mathrm{Si}_{3} \mathrm{~N}_{4}$ acquired with the MWNT tip is limited by the diameter of the MWNT. 
To further demonstrate that the high lateral resolution is indeed the result of the small diameter of the SWNT, the same SWNT probe was also employed to generate images of gold and iridium surfaces. As seen in Fig. $4 \mathrm{~b}$, a $2 \mathrm{~nm}$ thick gold film exhibits relatively large domains ranging in sizes from $30 \mathrm{~nm}$ to less than $10 \mathrm{~nm}$. It is important to point out that the smaller diameter of the SWNT tip is able to resolve greater lateral details of particles as seen by the variety of shapes in the AFM image. The same SWNT tip probe was also employed to examine a $2 \mathrm{~nm}$ thick iridium film on mica (Fig. 4c). The Ir grains appear to be rounder and even smaller in size (averaging $\sim 2-3 \mathrm{~nm}$ ) than those of the gold sample. Taken together, the results of this study demonstrate the power of CNT tip probes in terms of improving the capability of AFM, increasing lateral resolution by almost $5 \mathrm{X}$ over that of a conventional silicon AFM probe, and pointing toward perhaps a new and simple surface characterization technique that has immense potential in semiconductor manufacturing.

\section{Conclusion}

In summary, we have illustrated the stability and high lateral resolution capability of CNT probes for AFM. The MWNT probe shows no degradation in resolution after a long period of continuous scanning, and the SWNT tip probe is capable of lateral resolution as small as $2 \mathrm{~nm}$. The coupling of nanotube probes with AFM offers a simple and high throughput surface characterization technique ideal for applications in integrated circuit manufacturing.

\section{Acknowledgement}

This work was supported by the NASA Ames Nanotechnology program and the NASA$\mathrm{NCI}$ collaborative contract. Work by the personnel from Eloret Corporation was funded by a contract from NASA Ames to Eloret. Carl Larson of IBM Almaden Research Center is acknowledged for providing the patterns in Fig. 2. 


\section{References}

1. S. Iijima, Nature (London) 354, 56 (1991)

2. H. Dai, J.H. Hafner, A. G. Rinzler, D.T. Colber, and R.E. Smalley, Nature 384, 147150 (1996).

3. J.H. Hafner, C.L. Cheung, and C.M. Lieber, J. Am. Chem. Soc. 121, 9730-9731, (1999).

4. Y. Nakayama, H. Nishijima, S. Akita, K. Hohmura, S.H. Yoshimura, and K. Takeyasu, J. Vac. Sci. Technol. B, 18, 661 (2000).

5. R. M. D. Stevens, N.A. Frederick, B.L. Smith, D.E. Morse, G.D. Stucky, and P.K. Hansma, Nanotechnology, 11, 1 (2000).

6. W.L. Wu and W.E. Wallace, J. Vac. Sci. Technol. B, 16, 1963 (1998).

7. G.W. Reynolds and J.W. Taylor, J. Vac. Sci. Technol. B, 17, 2723 (1999).

8. Y.E. Strausser, M. Schroth, and J. Seeney III, J. Vac. Sci. Technol. A, 15, 1007 (1997).

9. R. Edrei, E.N. Shauly, and A. Hoffman, J. Vac. Sci. Technol. B, 18, 41 (2000).

10. R. Stevens, C. Nguyen, A. M. Cassell, L. Delzeit, M. Meyyappan and J. Han, App. Phys. Lett., in press.

11. A.M. Cassell, S. Verma, L. Delzeit, M. Meyyappan, and Jie Han, Langmuir, in press. 


\section{Figure Captions}

Figure 1. Force vs. distance plots for AFM cantilever with a single-walled carbon nanotube probe. Curve a: cantilever amplitude vs. Z-distance; Curve b: cantilever deflection vs. $Z$-distance. $Z=0$ denotes the surface.

Figure 2. AFM images of a $280 \mathrm{~nm}$ line/space array of polymeric photoresist $(300 \mathrm{~nm}$ thickness) on a silicon substrate acquired with a regular silicon probe (left image) and a multi-walled carbon nanotube probe (right image).

Figure 3. Time-lapse AFM images of a $2 \mathrm{~nm}$ thick silicon nitride film comparing MWNT and silicon probes (a) after a 15+ hrs. continuous scanning MWNT tip and (b) after a $12 \mathrm{hrs}$. continuous scanning regular silicon probe.

Figure 4. AFM images acquired with a single-walled carbon nanotube tip probe. (a) silicon nitride surface showing grain sizes as small as $3 \mathrm{~nm}$ (b) $2 \mathrm{~nm}$ thick gold film on mica showing grain sizes ranging from $30 \mathrm{~nm}$ to less than 10 $\mathrm{nm}$ and (c) $5 \mathrm{~nm}$ thick iridium film on mica showing Ir grain sizes about 2-3 nm. 


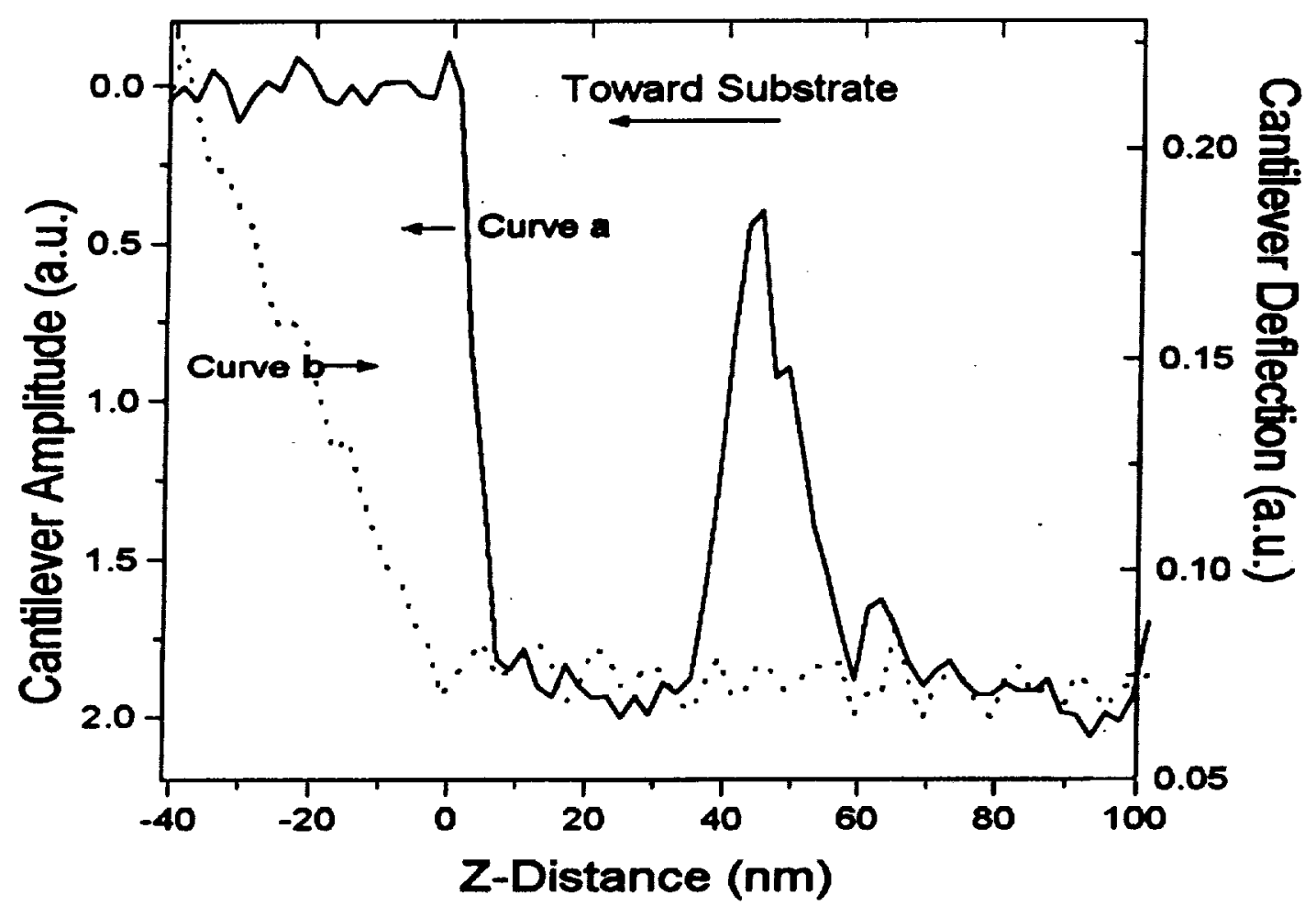




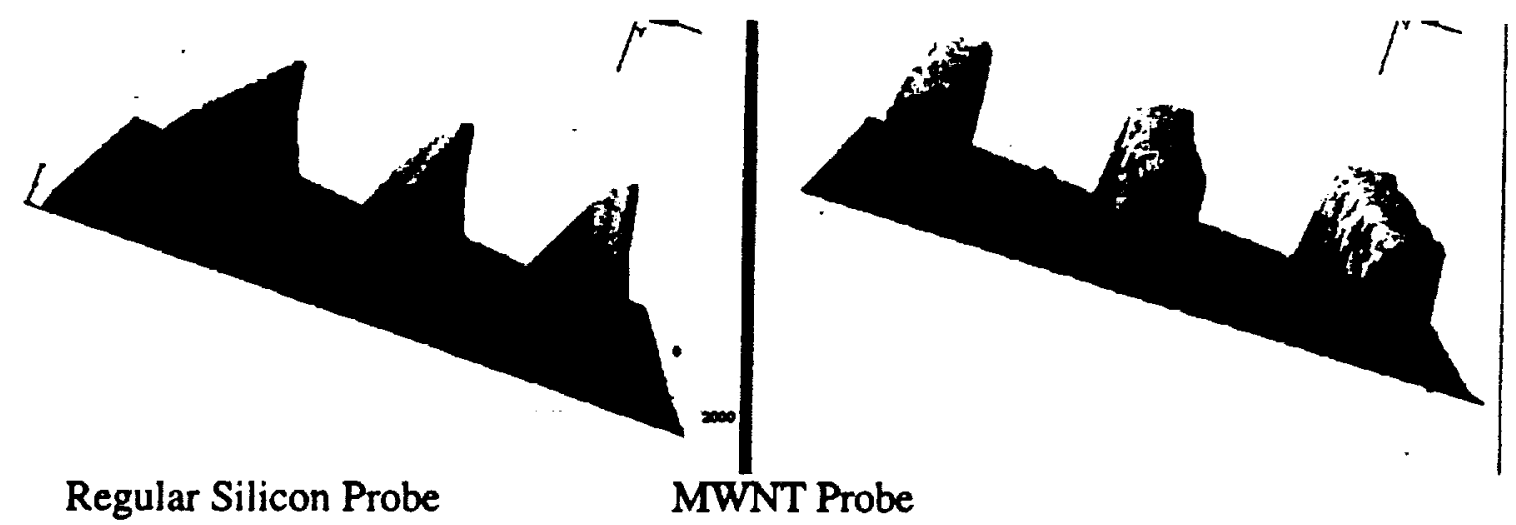



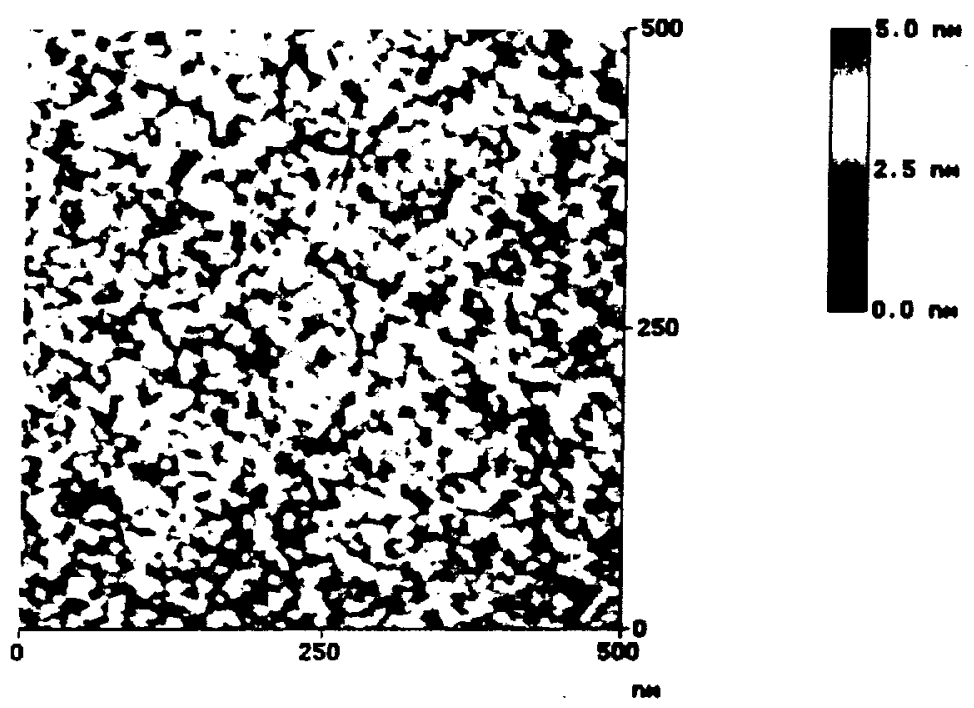

(a)
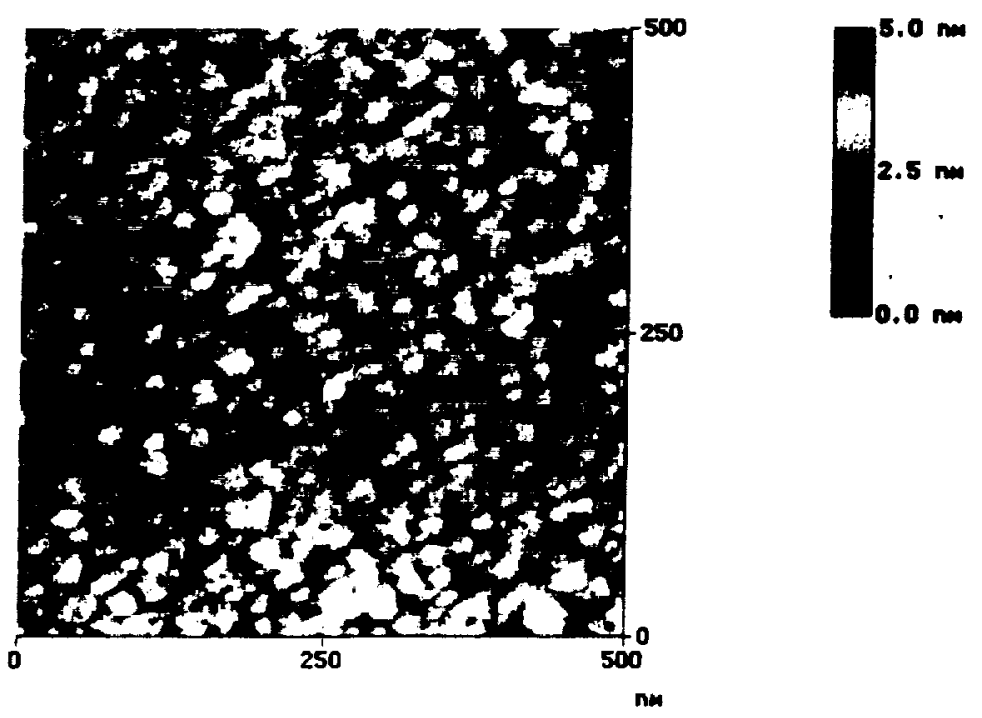

(b) 

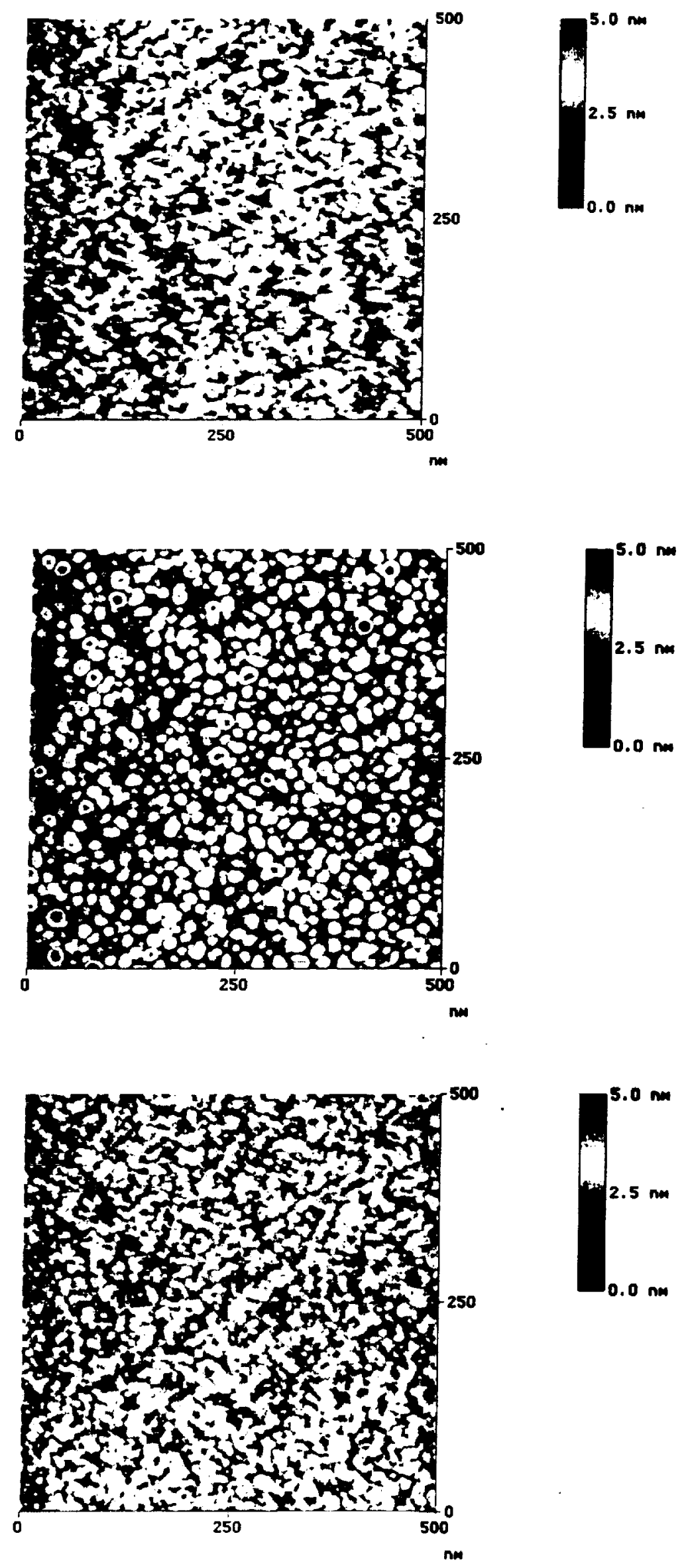